\title{
An equilibrium-conserving taxation scheme for income from capital
}

\author{
Jacques Tempere ${ }^{\mathrm{a}}$ \\ Theory of Quantum and Complex Systems, Universiteit Antwerpen, Universiteitsplein 1, 2610 Antwerpen, Belgium
}

Received 28 August 2017 / Received in final form 23 November 2017

Published online 14 February 2018

(C) The Author(s) 2018. This article is published with open access at Springerlink.com

\begin{abstract}
Under conditions of market equilibrium, the distribution of capital income follows a Pareto power law, with an exponent that characterizes the given equilibrium. Here, a simple taxation scheme is proposed such that the post-tax capital income distribution remains an equilibrium distribution, albeit with a different exponent. This taxation scheme is shown to be progressive, and its parameters can be simply derived from (i) the total amount of tax that will be levied, (ii) the threshold selected above which capital income will be taxed and (iii) the total amount of capital income. The latter can be obtained either by using Piketty's estimates of the capital/labor income ratio or by fitting the initial Pareto exponent. Both ways moreover provide a check on the amount of declared income from capital.
\end{abstract}

\section{Introduction}

The distribution of income has been studied for a long time in the economic literature, and has more recently become a topic of investigation for statistical physicists turning to econophysics [1-4]. The income distribution is characterized by a density function $f(x)$ such that $f(x) \mathrm{d} x$ is the number of individuals earning income between $x$ and $x+\mathrm{d} x$. From the empirical data obtained from tax records, two different regimes are readily distinguished. For income levels below a certain threshold $x_{c}$, the distribution follows and exponential (Boltzmann) law, $f(x) \propto$ $\exp (-x / \bar{x})$, whereas for income levels above $x_{c}$ the distribution is better fitted by a power law, $f(x) \propto x^{-\gamma}$. Note that for the bottom incomes, a deviation from the Boltzmann law is visible. This is due to redistribution (such as social security benefits) which lifts a certain amount of people above a poverty threshold $x_{\text {pov }}$.

For income above the poverty level but below $x_{c}$ the distribution is very well fitted by a Gibbs distribution $[5,6]$. Tax records that keep track of the source of income indicate that income in this regime is dominated by labor income (salaries and wages) [7]. In this regime, economic transactions can be modelled by additive processes $[4,6]$ : money exchanges hands between agents but the total amount of money is conserved over the transaction. For example, each month an employee gets a certain sum of money added to his account, and this sum is subtracted from the account of the employer's company. Using this principle of local money conservation, Dragulescu and Yakovenko [5] have shown that the equilibrium

\footnotetext{
${ }^{\mathrm{a}}$ e-mail: jacques.tempere@ua.ac.be
}

distribution of money over the agents involved in additive transactions follows a Boltzmann-Gibbs exponential distribution. Note that this is a strongly simplified model of economic activity: it is clear that in reality global money conservation is violated. Indeed, banks can issue (or recall) loans, thereby increasing (or decreasing) the total money supply.

This brings us to the higher incomes, $x>x_{c}$. As noted already by Pareto in 1897, these follow a power law [8]. Records that keep track of the source of income reveal that income in the power law regime is mainly capital income (rent, profits, interests, dividends, ... ) [9,10]. For these types of income, economic transactions are better modeled by multiplicative processes $[6,11,12]$. In contrast to a wage worker, a rentier expects that at each time step the money in his investment is multiplied by an interest factor. For such processes, it is $\log (x)$ rather than $x$ which is conserved locally, and this leads naturally to a power law rather than an exponential as equilibrium distribution [13-15].

The change from exponential regime to power-law regime occurs in a narrow interval $[16,17]$ around $x_{c}$, allowing to separate not only the income in two sources ( $M_{\text {lab }}$ from labor and $M_{\text {cap }}$ from capital), but also the population in two groups ( $N_{\text {lab }}$ and $N_{\text {cap }}$, respectively). Of course, these are not clearly delineated, and also people filing tax forms for income below $x_{c}$ have a portion of their income coming from return on capital. However, the problem can be greatly simplified by taking the main source of income to be the entire income. The value $x_{c}$ separating the exponential from the power-law regimes lies between three and four times the average income in the exponential part of the distribution [16-18]. 


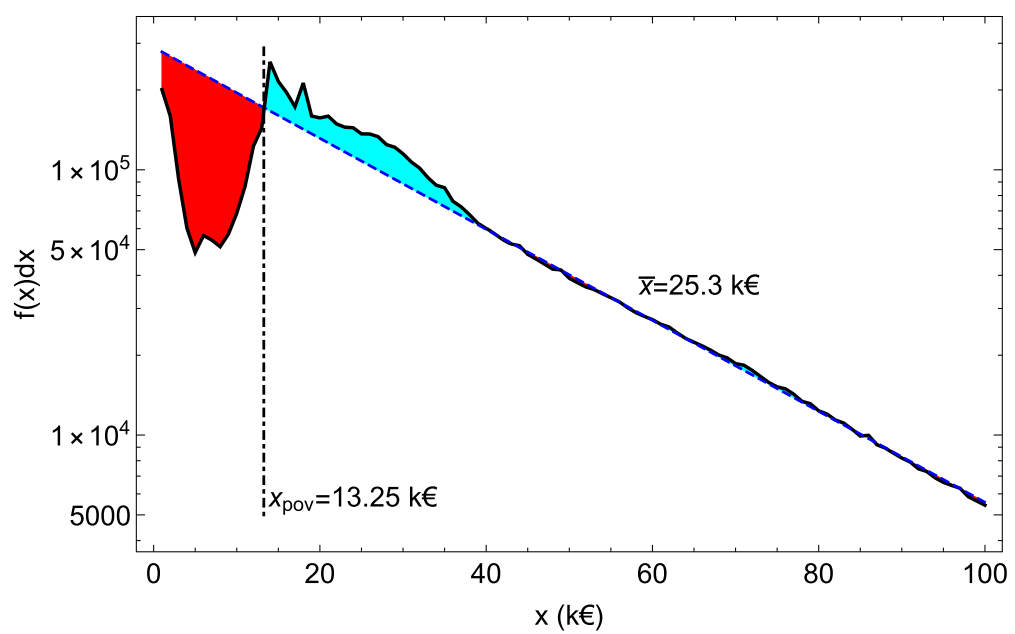

Fig. 1. The (annual) income distribution in Belgium in 2014 as obtained from personal tax records [21], in bins of $\mathrm{d} x=1 \mathrm{k} €$. The distribution follows a Boltzmann law (dashed line) except for the lowest income. Redistributive taxation lifts people with an income below $x_{\text {pov }}$ (area shaded in dark red) above this poverty threshold (area shaded in light blue).

There is a large body of work in the economics literature on optimal taxation of top incomes (for a recent review, see [19]), taking into account the effects of taxation on economic activity and feedback effects on the declared top incomes such as tax avoidance and compensation bargaining [20]). Here, I will not venture into the debate on the economics of taxation, but limit the scope to a straightforward mathematical question. The question that I wish to address is how to levy taxes such that the immediate after-tax income distribution remains in equilibrium (i.e. of Boltzmann type for $x<x_{c}$, and of Pareto type for $\left.x>x_{c}\right)$. The parameters $(\bar{x}$ and $\gamma)$ of the pretax and after-tax distributions will be different, reflecting the change from one equilibrium to another rather than a shift to a non-equilibrium distribution.

In Section 5 some arguments are presented in favor of equilibrium-to-equilibrium taxation. The main idea is that it corresponds to a shift from one Pareto efficient state to another. In contrast, a taxation scheme that results in an out-of-equilibrium after-tax income distribution corresponds to a move away from Pareto efficiency and hence away from an optimal allocation of resources. Of course, whether any scheme is just or desirable is well beyond the scope of this paper. Nevertheless, given the current debate taking place (in the USA, the UK and the EU) of how to tax the rich, I believe the results presented here can contribute to an informed discussion.

\section{Capital and labor income for Belgium}

To illustrate the results with numbers, I use my home country of Belgium as an example, taking $x_{c} \approx 100 \mathrm{k} €$. There were $N_{\text {tot }}=6.26 \times 10^{6}$ people filing a non-zero income tax record in Belgium in 2014, the latest year with complete information [21]. Of these, $N_{\text {cap }}=1.73 \times 10^{5}$ (or $2.8 \%$ of the total) indicate an income above $x_{c}$. The remaining $N_{\text {lab }}=6.09 \times 10^{6}$ are in the exponential regime and represent a cumulative income of $M_{\mathrm{lab}}=170.6 \mathrm{G} €$.
As seen in Figure 1, there is a strong deviation of the exponential regime for income levels below $x_{\mathrm{pov}}=13.25 \mathrm{k} €$ : this is the poverty threshold. People below this threshold receive social security benefits lifting them to roughly the threshold level and slightly above.

Estimating the amount of capital income $M_{\text {cap }}$ for Belgium is difficult to do based on tax forms. The reason is that not all sources of capital income need to be declared in Belgium: for instance, income from renting out apartments or offices is not declared. Piketty [22] provides an alternative way to estimate $M_{\text {cap }}$ from $M_{\text {lab }}$. His study of the historical ratio between capital income and labor income for developed economies shows that this income has its own slow dynamics over time. Currently, the capital share of income in rich countries stands at $25-30 \%$ of national income. The dynamics of the capital/labor income ratio is slow enough such that the income distribution is close to equilibrium at any time. Taking the above-mentioned capital share of the national income into account results in an estimate of $M_{\text {cap }} \approx 60 \mathrm{G} €$.

The additive class, $x_{\mathrm{pov}}<x<x_{c}$ is subject to the (normalized) Boltzmann-Gibbs distribution

$$
f_{\text {lab }}(x)=\frac{N_{\text {lab }}}{\bar{x}} \exp (-x / \bar{x})
$$

with $\bar{x}=M_{\text {lab }} / N_{\text {lab }}$ the average income in this class.

The multiplicative class is subject to the power law distribution

$$
f_{\text {cap }}\left(x>x_{c}\right)=\frac{(\gamma-1) N_{\text {cap }}}{x_{c}}\left(x / x_{c}\right)^{-\gamma} .
$$

The Pareto parameter $\gamma$ is fixed by $M_{\text {cap }}$ through the normalization $M_{\text {cap }}=\int_{x_{c}}^{\infty} x f_{\text {cap }}(x) \mathrm{d} x$ by

$$
\gamma=\frac{2 M_{\text {cap }}-N_{\text {cap }} x_{c}}{M_{\text {cap }}-N_{\text {cap }} x_{c}} .
$$


Table 1. Data for US income distribution, tax year 2015, from [24]. The first column shows the income bracket, the second column is the number of tax returns in this bracket, and the third column is the total income of all returns in this bracket.

\begin{tabular}{lll}
\hline Income bin $(\mathrm{k} \$)$ & \#Returns & Total amount $(\mathrm{G} \$)$ \\
\hline$[10,15[$ & 12219480 & 152.752468 \\
{$[15,20[$} & 11228447 & 195.857688 \\
{$[20,25[$} & 9981450 & 224.230854 \\
{$[25,30[$} & 8832875 & 242.572775 \\
{$[30,40[$} & 14913880 & 519.525813 \\
{$[40,50[$} & 11625418 & 520.845982 \\
{$[50,75[$} & 19980117 & 122.8299087 \\
{$[75,100[$} & 12821791 & 1111.174843 \\
{$[100,200[$} & 18532593 & 2506.497828 \\
{$[200,500[$} & 5428176 & 1546.515483 \\
{$[500,1000[$} & 884335 & 597.676645 \\
{$[1000,1500[$} & 195905 & 236.499605 \\
{$[1500,2000[$} & 79971 & 137.686352 \\
{$[2000,5000[$} & 116718 & 346.864436 \\
{$[5000,10000[$} & 28680 & 195.661353 \\
\hline
\end{tabular}

This restricts $\gamma>2$, since $M_{\text {cap }}>N_{\text {cap }} x_{c}$. The estimate $M_{\text {cap }}=60 \mathrm{G} €$ based on Piketty's observations corresponds to $\gamma=2.4$. This value is in agreement with the estimate of $\gamma \approx 2.5$ obtained by Silva and Yakovenko [18].

Equation (3) can also be inverted, so that an empirical fit yielding $\gamma$ (in combination with $x_{c}$ and $N_{\text {cap }}$ ) may be used to estimate the total amount of income from capital. Detailed data for the high-income distribution is not publicly available in Belgium, and as mentioned above, exemption of some capital income sources in Belgium complicates data finding. However, for countries with an obligation to declare all capital income, equation (3) could be used to estimate the amount of undeclared income and hence the level of tax evasion by rentiers. A similar proposal, based on deviations from the Pareto distribution, was introduced to estimate the size of shadow banking [23].

\section{Capital and labor income for the USA}

As in Belgium, income from capital is mostly exempt from taxation, it is illustrative to show an example where also the higher income part of the income distribution is available. For this purpose, we use the tax data publicly available [24] for the USA, for the fiscal year 2015. The data is listed in Table 1. Although the data bins are more coarse grained than the data for Belgian tax returns, the data extends to much larger incomes.

Figure 2 shows the resulting income distribution on a log-log plot. The lower income part, interpreted as income from labor, is fitted by an exponential, as in the case of the Belgian data, with now $\bar{x}=47 \mathrm{k} \$$. The higher income part (for which data is not available for Belgium) is fitted by a Pareto power law, with $\gamma=2.48$, and $N_{\text {cap }}=3.8 \times 10^{6}$. In the present scheme, we interpret this part as income derived mainly from capital. The two curves intersect at $x_{c}=230 \mathrm{k} \$$.
This allows to calculate the income from capital as derived from the fit,

$$
M_{\text {cap }}=\frac{\gamma-1}{\gamma-2} N_{\text {cap }} x_{c}
$$

which evaluates to $2.8 \mathrm{~T} \$$. This value derived from the fit compares well with the total income above $x_{c}$ from the returns, $2.2 \mathrm{~T} \$$. The value derived from the fit indicates a capital share of income of $28 \%$. The value found by the present method agrees with Piketty's result [22] of $29 \%$ (for 2010), which was obtained in a quite different way, namely based on the national accounts for the USA.

\section{Taxing capital income}

Suppose one wants to levy taxes to raise a given amount $\Delta M$ of money. For example, to bring all the poor to a minimum wage of $x_{\text {pov }}$, one would need

$$
\begin{aligned}
\Delta M & =\int_{0}^{x_{c}}\left(x_{c}-x\right) f_{\mathrm{lab}}(x) \mathrm{d} x \\
& =M_{\mathrm{lab}}\left[\left(x_{\mathrm{pov}} / \bar{x}\right)-\left(1-e^{-x_{\mathrm{pov}} / \bar{x}}\right)\right] .
\end{aligned}
$$

Using the numbers listed above for Belgium, the topping up of all lower incomes to $x_{\text {pov }}$ would require $16.4 \mathrm{G} €$.

Suppose moreover that one wants to obtain the amount of $\Delta M$ by taxing capital income in such a way that the after-tax capital income distribution follows again a Pareto law. The post-tax power law distribution necessarily has a different exponent $\eta>\gamma$, given by

$$
\eta=\frac{2\left(M_{\text {cap }}-\Delta M\right)-N_{\text {cap }} x_{c}}{M_{\text {cap }}-\Delta M-N_{\text {cap }} x_{c}} .
$$

In our Belgian example, this would mean a change from $\gamma=2.41$ to $\eta=2.66$. To describe the taxation scheme, we introduce a function $X(x)$ that gives the post-tax net income $X$ as a function of the pre-tax income $x$. The distribution of post-tax income is denoted by

$$
f_{\text {cap }}^{\text {post-tax }}(X)=\frac{(\eta-1) N_{\text {cap }}}{x_{c}}\left(X / x_{c}\right)^{-\eta} .
$$

This distribution has to obey

$$
f_{\text {cap }}^{\text {post-tax }}(X) \mathrm{d} X=f_{\text {cap }}[x(X)] \mathrm{d} x .
$$

Substituting the Pareto distributions in the above equation yields a differential equation for $\mathrm{X}(\mathrm{x})$,

$$
\frac{(\eta-1)}{x_{c}^{1-\eta}} X^{-\eta}(x) \frac{\mathrm{d} X}{\mathrm{~d} x}=\frac{(\gamma-1)}{x_{c}^{1-\gamma}} x^{-\gamma} .
$$

It solution depends on the parameter

$$
\tau=\frac{\gamma-1}{\eta-1}
$$




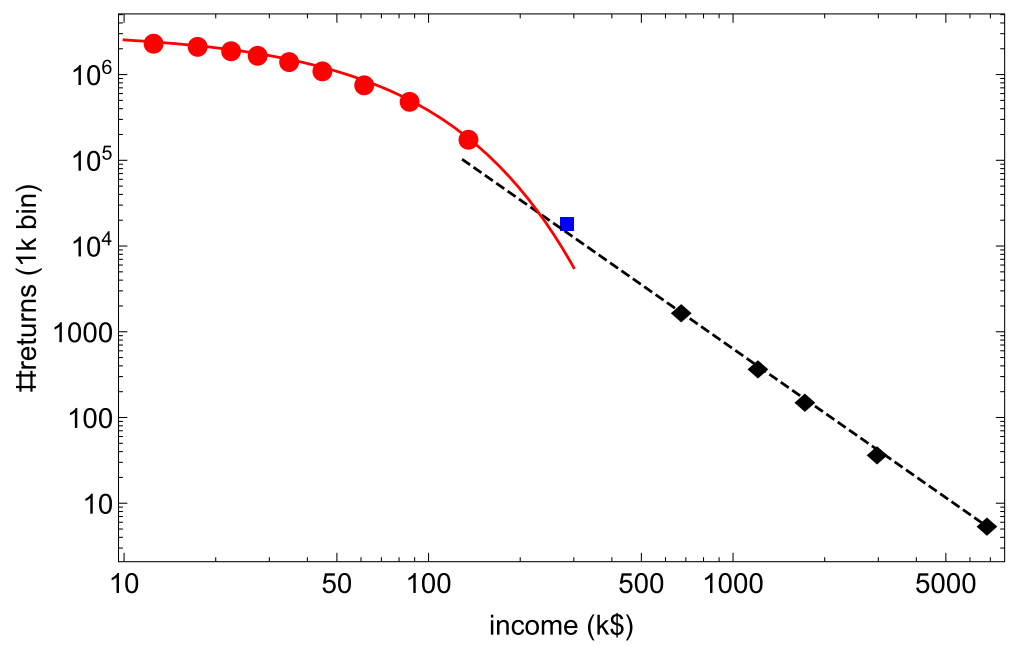

Fig. 2. The income distribution for the USA is shown for the fiscal year 2015. The (red) full curve is a fit with an Boltzmann exponential for the labor part of the distribution, using the data points shown as (red) circles. The (black) dashed line is a fit with a Pareto power law for the capital part of the income distribution, using the data points shown as (black) diamonds.

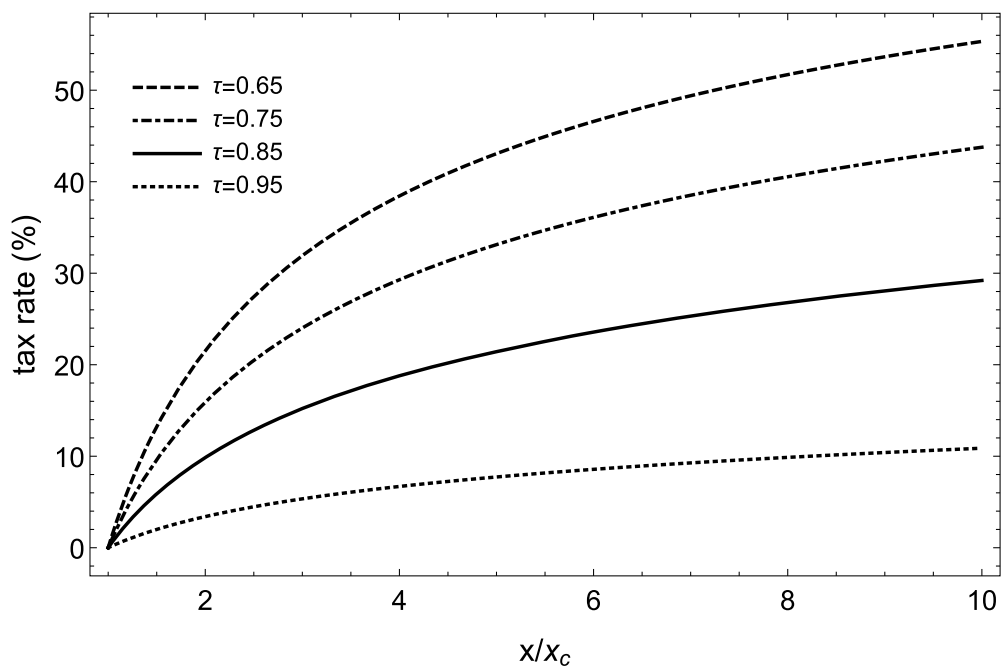

Fig. 3. Taxation levels as a function of capital income, for different values of the parameter $\tau$, which is fixed by the amount of $\operatorname{tax} \Delta M$ that will be levied from the total capital income $M_{\text {cap }}$. The tax rate is progressive and taxation starts at the income level $x_{c}$ separating the Boltzmann-type labor income distribution from the Pareto-type capital income distribution.

and is given by

$$
X(x)=x_{c}^{1-\tau} x^{\tau}
$$

As $\tau<1$, this solution corresponds to a weighted geometric averaging between the capital income and the threshold $x_{c}$ where main income switches from additive to multiplicative. For a pre-tax income $x$ the corresponding tax rate $T(x)=(x-X) / x$ is given by

$$
T(x)=1-\left(x_{c} / x\right)^{1-\tau} .
$$

Taking our example $\tau=0.85$, the resulting tax rate is shown as the full curve in Figure 3. A tax that rises proportionally to income is called "proportional" or "flat" (since the tax rate is constant). A tax that rises more than in proportion of income is called "progressive". From Figure 3 it is clear that the proposed taxation scheme for capital income is progressive. Whereas for a capital income of $120 \mathrm{k} €$ (slightly above the threshold when one can be called a rentier or "rich" in Belgium) the tax rate is about $3 \%$, at $200 \mathrm{k} €$ it has risen to $10 \%$, doubling again to $20 \%$ for $500 \mathrm{k} €$.

\section{Discussion and conclusions}

Equations (7), (11) and (13) represent a simple taxation scheme that preserves the power law nature of capital income. In order to implement it, policy makers need to select a threshold $x_{c}$ of income from capital above which the tax is levied, and choose a value of $\tau$, or equivalently, an amount $\Delta M$ of money that the tax should raise. The 
basic idea behind preserving the power law is that this law represents a distribution in which the market is in equilibrium.

Why might one advocate a taxation that results in a power-law post-tax distribution of capital income? The reasoning behind why such scheme can be desirable consists of two parts.

The first part of the reasoning concerns the meaning of "equilibrium" in capital markets. Models based on interacting economic agents have been used extensively to understand the occurrence of power law tails both in wealth distributions and capital return distributions in financial markets (for a review, see [25]). As the time variation of the capital returns are proportional to capital itself, this leads to multiplicative stochastic models [15] which in turn lead to the observed power-law behavior. The interacting "gas" of agents is described by a Langevin-type equation [26]. The ensuing dynamics are such that any initial distribution of capital among the agents will first undergo a transient relaxation, after which it settles into an equilibrium characterized by a power law-distribution. It is in this "thermodynamic" sense that I claim the market for capital returns (which I assume will be mainly the financial market) is in equilibrium when a Pareto distribution is present $[15,27]$. Although many trades are still taking place, the overall distribution of capital returns has become stationary (barring external shocks). There are many different equilibria possible, characterized by the power law exponent.

The second part of the reasoning is that there is a relation between market equilibrium and market efficiency. This relation is known as the first fundamental theorem of welfare economics, and states that the equilibrium situation produced by a competitive, free market is Pareto efficient. Pareto efficiency is an outcome in which there is optimal allocation of resources - it is not possible to reallocate resources without making at least one of the agents worse off. This type of efficiency is considered as a minimal requirement for a socially desirable distribution of resources (see for example [28]). Although this assumption can certainly be criticized, much of theoretical and applied welfare economics is based on it.

Bringing both parts together, the conclusion is that if market efficiency is a desirable outcome, then a scheme that results in a market equilibrium is preferable.

In addition, the second fundamental theorem of welfare economics states that a social planner can select the most suitable Pareto efficient outcome out of the many possible equilibria, by performing lump sum transfers. In this context, the current proposal for taxation is precisely a way to organise a transfer that links one equilibrium for capital markets to another.

What if one would use the same logic to labor income? Changing one Boltzmann distribution into another only requires a scale change $x \rightarrow \alpha x$ where $\alpha=1-\Delta M / M_{\text {lab }}$. This corresponds to a proportional tax system (a "flat tax", such as a fixed sales tax). This is not always seen as the socially most desirable outcome as it penalizes the low-income segment of the population, who have less disposable income. In essence, the current proposal represents a flat tax on $\log (x)$, modified by the presence of a the threshold $x_{c}$. Regardless of the desirability debate, it is clear that proponents of a flat tax for labor income who base their arguments on market efficiency, should then logically advocate the current progressive tax on capital income. Another commonly encountered argument for a flat tax is its simplicity. In this respect, the proposal for a progressive capital income taxation put forward in this paper offers a scheme which, at least to a physicist, is of similar simplicity.

Open Access This is an open access article distributed under the terms of the Creative Commons Attribution License (http://creativecommons.org/licenses/by/4.0), which permits unrestricted use, distribution, and reproduction in any medium, provided the original work is properly cited.

\section{References}

1. A. Chatterjee, S. Yarlagadda, B.K. Chakrabarti (eds.), Econophysics of wealth distributions (Springer Verlag, Milan, 2005)

2. A. Chatterjee, B.K. Chakrabarti, Eur. Phys. J. B 60, 135 (2007)

3. V.M. Yakovenko, J. Barkley Rosser, Rev. Mod. Phys. 81, 1703 (2009)

4. B.K. Chakrabarti, A. Chakraborti, S.R. Chakravarti, A. Chatterjee, Econophysics of income wealth distributions (Cambridge University Press, Cambridge, 2013)

5. A.A. Dragulescu, V.M. Yakovenko, Eur. Phys. J. B 17, $723(2000)$

6. S. Ispolatov, P.L. Krapivsky, S. Redner, Eur. Phys. J. B 2, 267 (1998)

7. G. Willis, J. Mimkes, arXiv:cond-mat/0406694

8. V. Pareto, Cours d'Economie Politique, edited by F. Rouge (Librairie de l'Université, Lausanne, 1897)

9. F. Clementi, M. Gallegati, Income inequality dynamics: evidence from a pool of major industrialized countries, in Talk at the International Workshop of Econophysics of Wealth Distributions, Kolkata, March 15-19, 2005. Retrieved from http://www.saha.ac.in/cmp/econophysics/abstracts.html

10. T.L. Hungerford, J. Curr. Issues Finance Bus. Econ. Retrieved from http://taxprof.typepad.com/files/crs1.pdf

11. M. Levy, H. Levy, Rev. Econ. Stat. 85, 709 (2003)

12. Y. Fujiwara, W. Souma, H. Aoyama, T. Kaizoji, M. Aoki, Physica A 321, 598 (2003)

13. M. Levy, S. Solomon, Int. J. Mod. Phys. C 7, 595 (1996)

14. O. Biham, O. Malcai, M. Levy, S. Solomon, Phys. Rev. E 58, 1352 (1998)

15. P. Richmond, S. Solomon, Int. J. Mod. Phys. C 12, 333 (2001)

16. A.A. Dragulescu, V.M. Yakovenko, Physica A 299, 213 (2001)

17. A.A. Dragulescu, V.M. Yakovenko, Statistical mechanics of money, income, and wealth: a short survey, in Modeling of complex systems: seventh granada lectures, edited by P.L. Garrido, J. Marro (American Institute of Physics, AIP Conference Proceedings, Melville, NY, 2003) Vol. 661

18. A.C. Silva, V.M. Yakovenko, Europhys. Lett. 69, 304 (2005) 
19. E. Saez, J. Slemrod, S.H. Giertz, J. Econ. Lit. 50, 3 (2012)

20. T. Piketty, E. Saez, S. Stantcheva, Am. Econ. J. 6, 230 (2014)

21. Data source: Algemene Directie Statistiek - Statistics Belgium, Fiscale inkomens, http://statbel.fgov.be/nl/ modules/publications/statistiques/arbeidsmarkt_levenso mstandigheden/Statistique_fiscale_des_revenus.jsp (retrieved on July 1st, 2017)

22. T. Piketty, Capital in the twenty-first century (Harvard University Press, Cambridge, USA, 2014) Translation A. Goldhammer
23. D. Fiaschi, I. Kondor, M. Marsili, V. Volpati, PLoS ONE 9, e94237 (2014)

24. Data source: https://www.irs.gov/statistics retrieved on October 25th, 2017

25. M. Levy, H. Levy, S. Solomon, Microscopic simulation of financial markets (Academic Press, New York, 2000)

26. P. Richmond, Eur. Phys. J. B 20, 523 (2001)

27. S. Solomon, P. Richmond, Eur. Phys. J. B 27, 257 (2002)

28. N. Barr, Economics of the welfare state, 5th edn. (Oxford University Press, Oxford, 2012) 